\title{
Time course and frequency specificity of sub-cortical plasticity in adults following acute unilateral deprivation
}

\author{
Hannah Brotherton \\ Manchester Centre for Audiology and Deafness, University of Manchester, Manchester Academic Health Science Centre, Manchester, M13 \\ 9PL, United Kingdom \\ hannah.brotherton@manchester.ac.uk
}

\section{Christopher J Plack}

Manchester Centre for Audiology and Deafness, University of Manchester, Manchester Academic Health Science Centre, Manchester, M13 9PL, United Kingdom

chris.plack@manchester.ac.uk

Department of Psychology, Lancaster University, Lancaster, LA1 4YF, United Kingdom

Roland Schaette

Ear Institute, University College London, London, WC1X 8EE, United Kingdom

r.schaette@ucl.ac.uk

Kevin J Munro

Manchester Centre for Audiology and Deafness, University of Manchester, Manchester Academic Health Science Centre, Manchester, M13 9PL, United Kingdom

Central Manchester University Hospitals NHS Foundation Trust, Manchester Academic Health Science Centre, Manchester, M13 9WK, United Kingdom

kevin.j.munro@manchester.ac.uk

Corresponding author: hannah.brotherton@manchester.ac.uk ${ }^{1}$

\footnotetext{
${ }^{1}$ Present address: Department of Communication Sciences \& Disorders, University of South Florida, 4202 E. Fowler Avenue, PCD1017, Tampa, FL 33620-8200
} 


\section{ABSTRACT}

2 Auditory deprivation and stimulation can change the threshold of the acoustic reflex, but the mechanisms underlying these changes remain largely unknown. In order to elucidate the mechanism, we sought to characterize the time-course as well as the frequency specificity of changes in acoustic reflex thresholds (ARTs). In addition, we compared ipsilateral and contralateral measurements because the pattern of findings may shed light on the anatomical location of the change in neural gain. Twenty-four normal-hearing adults wore an earplug continuously in one ear for six days. We measured ipsilateral and contralateral ARTs in both ears on six occasions (baseline, after 2, 4 and 6 days of earplug use, and 4 and 24 hours after earplug removal), using pure tones at $0.5,1,2$ and $4 \mathrm{kHz}$ and a broadband noise stimulus, and an experimenter-blinded design. We found that ipsi- as well as contralateral ARTs were obtained at a lower sound pressure level after earplug use, but only when the reflex was elicited by stimulating the treatment ear. Changes in contralateral ARTs were not the same as changes in ipsilateral ARTs when the stimulus was presented to the control ear. Changes in ARTs were present after 2 days of earplug use, and reached statistical significance after 4 days, when the ipsilateral and contralateral ARTs were measured in the treatment ear. The greatest changes in ARTs occurred at 2 and $4 \mathrm{kHz}$, the frequencies most attenuated by the earplug. After removal of the earplug, ARTs started to return to baseline relatively quickly, and were not significantly different from baseline by 4-24 hours. There was a trend for the recovery to occur quicker than the onset. The changes in ARTs are consistent with a frequency-specific gain control mechanism operating around the level of the ventral cochlear nucleus in the treatment ear, on a time scale of hours to days. These findings, specifically the time course of change, could be applicable to other sensory systems, which have also shown evidence of a neural gain control mechanism.

Abbreviations: (ABR), Auditory brainstem response; (ART), Acoustic reflex threshold; (BBN), Broadband noise; (DCN), Dorsal cochlear nucleus; (IHC), inner hair cells; (SOC), superior olivary complex; (VCN), ventral cochlear nucleus. 
40 Short-term auditory deprivation can modify auditory physiology. In humans, this has been evident through changes in the acoustic reflex threshold (ART, the threshold sound level for a brainstem reflex that involves the bilateral contraction of the middle ear muscles) after auditory deprivation. When one ear was deprived from input by using an earplug to induce a mild to moderate hearing loss for several days, the ART was decreased in the treatment ear (Maslin et al., 2013; Munro et al., 2009; Munro et al., 2014). Moreover, additional stimulation through low-gain hearing aids has been shown to increase the ART (Munro et al., 2013), suggesting that neural response gain in the auditory brainstem might be increased or decreased, respectively, in an activity-dependent fashion (Schaette and Kempter, 2006; 2009).

Enhanced neural gain is hypothesized to be a potential mechanism in the development of tinnitus and hyperacusis (Auerbach et al., 2014; Brotherton et al., 2015; Eggermont et al., 2014; Schaette et al., 2006), two debilitating auditory conditions that affect a large proportion of the population (Andersson et al., 2002; Dawes et al., 2014). Since plugging one ear for several days can also induce the perception of phantom sounds (Schaette et al., 2012) and increase the perceived loudness of sounds (Formby et al., 2003; Munro et al., 2014), the changes caused by auditory deprivation might also be involved in the generation of tinnitus and hyperacusis. A detailed characterization of the gain mechanism underlying changes in ART could therefore provide insights into how tinnitus and hyperacusis are generated.

61 Changes in ARTs after deprivation or stimulation have been measured in humans in a series of studies (see Table I). A detailed characterization of time course and frequency-specificity of the effects are desirable, as the information available from previous studies is incomplete 
64 in these respects. Also, the location within the auditory pathway where changes in gain might be generated has still to be identified.

66

67 The first area of interest concerns the time course of changes in the neural gain mechanism following auditory deprivation. Most studies have investigated changes in ART after 7 days of continuous earplug use (Maslin et al., 2013; Munro et al., 2009; Munro et al., 2014). Only two studies have investigated a change in ART earlier than 7 days. Decker et al. (1981) investigated the ART following 10, 20 and 30 hours of unilateral earplug use. The authors observed a significant decrease in the mean ART at $2 \mathrm{kHz}$ after 10, 20 and 30 hours of unilateral earplug use. There was no difference in the mean change of ART across the different durations of deprivation. Changes in ART after 3-5 days of treatment have also been reported following acoustic stimulation (Munro and Merrett, 2013). Munro et al. (2013) investigated the ART following 3 and 5 days of hearing aid use in one ear. The authors reported an increase in the ART relative to baseline in an ear fitted with a hearing aid, and a reduction in the ART in the control ear, 3-5 days after augmented auditory stimulation. However, as the authors did not measure ARTs earlier than 3 days, it is unclear if changes occurred on a shorter time scale. Similarly, little is known about the time course of recovery following earplug removal. Munro et al. (2009) were able to demonstrate a return of ART values to baseline level 7 days after earplug removal, but earlier time points were not studied. In a further study, Munro et al. (2014) demonstrated that most of the asymmetry between the treatment and control ears had disappeared 1 day after earplug removal. To the authors' knowledge, there are no studies that have investigated a change in neural gain in normal hearing listeners less than 24 hours after earplug removal. 
Focusing on the second area of interest, much uncertainty exists about the relation between the frequency-range of elevated audiometric thresholds and enhanced neural gain. For example, does the compensatory change in neural gain occur in the frequency region of hearing loss? If so, it would be expected that short-term auditory deprivation would also have most effect on the ART at the frequencies attenuated by the earplug. Munro et al. (2009) limited ART measurements to 2 and $4 \mathrm{kHz}$, which received a similar level of attenuation by the earplug, and showed similar changes at both frequencies. Munro et al. (2013) investigated 0.5 and $2 \mathrm{kHz}$ and Maslin et al. (2013) investigated 0.5 and $4 \mathrm{kHz}$, and both studies found a larger change from baseline in ART at the higher frequency (where most earplug attenuation occurred), but the difference was not significant. Only one study in humans has attempted to investigate the change in ART at more than two frequencies. Decker et al. (1981) measured ARTs for $0.5,1$, and $2 \mathrm{kHz}$ tones. They reported a significant reduction in ART in the treatment ear at $2 \mathrm{kHz}$ in normal hearing listeners after 10, 20 and 30 hours of unilateral earplug use. For the lower frequencies $(0.5$ and $1 \mathrm{kHz})$, a similar trend was reported, but the changes did not achieve significance. A comparison between the frequencies was not performed. Although inconclusive, due to lack of significance, these findings suggest that the greatest change in neural gain may occur at frequencies most affected by the deprivation treatment. A frequency-specific mechanism would be consistent with tinnitus, which has shown to display a dominant pitch around the frequency range of the hearing loss (König et al., 2006; Sereda et al., 2011), whilst hyperacusis generally shows a change in loudness judgments across a range of frequencies (Anari et al., 1999, Sheldrake et al., 2015).

The pathway of the acoustic reflex arc involves the primary afferent fibers from the inner hair cells (IHCs) innervating the ventral cochlear nucleus (VCN), with projections from the VCN innervating the superior olivary complex (SOC) and projecting through the ipsilateral facial 
nerve nucleus to the ipsilateral stapedius muscle. The ipsilateral SOC also projects to the

114 contralateral facial nerve nucleus, which projects to the contralateral stapedial muscle (Lee et al., 2006). Therefore, the changes in the ART following unilateral earplug use (Maslin et al., 2013; Munro et al., 2009; Munro et al., 2014) or unilateral hearing aid use (Munro et al.,

117 2013) suggest that the gain mechanism operates within the subcortical auditory system. A change in neural gain in the cochlear nucleus after earplug deprivation would be consistent with a change in the ART. However, the efferent system has been shown to modulate the acoustic reflex (Campo et al., 2007). Therefore, changes in neural activity in the efferent pathway could present themselves as a change in the ART. If the efferent pathway were involved in changes in the ART after earplug use, it would be expected that following unilateral earplug use, a similar change in ART would be observed when the reflex is measured in the treatment ear, regardless of whether the reflex is elicited through ipsilateral or contralateral stimulation.

The present study extended the work of Munro et al. (2009), Maslin et al. (2013) and Munro et al. (2014) by investigating: (1) the time course of changes in ARTs following auditory deprivation; (2) the changes in ARTs for a range of frequencies, and (3) the location of change along the auditory pathway. The first and seconds aims were addressed using ipsilateral ARTs, while the latter aim was investigated by comparing the change in ipsilateral

132 ARTs with the change in contralateral ARTs. ARTs were measured using pure tones with a range of different frequencies to elicit the reflex over 6 days of continuous unilateral earplug use. Based on the trends from previous ART studies (Maslin et al., 2013; Munro et al., 2013; Munro et al., 2014) it was hypothesized that the reduction in ARTs would be greatest at the frequencies most attenuated by the earplug. Moreover, based on the results of Munro et al. (2013) it was hypothesized that the onset of the reduction in ARTs would occur earlier than 7 
138 days. Based on the findings of Munro et al. (2014) it was hypothesized that complete 139 recovery to baseline would occur 24 hours after the removal of the earplug. Finally, ARTs 140 were measured using both ipsi- and contralateral ARTs because the pattern of findings may 141 shed light on the anatomical location of the change in neural gain. Specifically, we 142 hypothesized that if the change in neural gain occurred at the level of the VCN, a reduction of 143 the ARTs would be observed in each ear when the treatment ear is stimulated to elicit the 144 reflex. 


\section{METHODS}

\subsection{Participants}

149 Based on the results of a pilot study, showing an asymmetry of $4.5 \mathrm{~dB}$ (s.d. \pm 6 ) between the ears at $2 \mathrm{kHz}$ following 2 days of unilateral earplug use, we calculated that 16 participants would be required to reach a power of $80 \%$ for a within-subjects factor for a two-tailed paired-samples t-test at 5\% significance level. Twenty-eight consenting volunteers (20 female and eight males; median age 21 years; participants were all between 18 and 28 years except two who were 31 and 59 years) were recruited to the study, to allow for attrition and a smaller than expected effect size. The study received ethics approval from the University of Manchester (Ref: 13183).

All participants were screened for normal-hearing sensitivity (i.e. thresholds $<20 \mathrm{~dB}$ HL from 0.25 to $8 \mathrm{kHz}$ and no inter-aural asymmetry $>10 \mathrm{~dB}$ at any frequency) and normal middle ear function on tympanometry (middle ear pressure +50 to -50 daPa, middle ear compliance 0.3 to $1.5 \mathrm{~cm}^{3}$ ). Four participants were excluded from analysis because of incomplete data: one participant did not take part in all test sessions due to time constraints and it was not possible to measure the ART at most frequencies in the remaining three participants. The excluded data were from younger participants. One additional participant was unable to complete the study due to cerumen impaction. Evidence of cerumen impaction removed blinding and prevented testing, therefore the data from this participant was not included in the final analysis of the present study. As this participant did not complete the study, they were not considered as part of 28 participants that completed the study. 


\subsection{Noise-attenuating earplugs}

171 The 24 participants who completed the study were fitted monaurally (11 left ear, 13 right ear) with a reusable Mack's silicone earplug (McKeon Products, United States) and instructed to wear it continuously for 6 days. As a pilot study had shown that 2 days of unilateral earplug use induced a change in the ART, we therefore investigated the time course of change in ART at equal intervals at day 0,2, 4 and 6 of earplug use. ART measurements on day 6 allowed a comparison with the findings from previous ART studies (Munro et al., 2009; Munro et al., 2014). To investigate the recovery of ART towards baseline levels after earplug removal, we measured the ART 4 and 24 hours after the removal of the earplug. The 24 hour time-point was chosen to allow a direct comparison of the findings with the results of Munro et al. (2014).

181

Sound attenuation levels (i.e., the difference in ear-canal sound level with and without the earplug in situ) were measured using a clinical probe-microphone system (Verifit@). A calibrated probe microphone was inserted into the ear canal and the response to a $65 \mathrm{~dB}$ sound pressure level (SPL) pink noise signal was measured before and after the insertion of the earplug. The measures were made three times after the participant removed and refitted the earplug into each ear. The attenuation values for each of the three fittings (from the treatment ear) and the mean attenuation values across the three fittings are shown in Fig 1. The average attenuation values were $9-16 \mathrm{~dB}$ at $0.5-1 \mathrm{kHz}$ and $24-30 \mathrm{~dB}$ at $2-4 \mathrm{kHz}$.

Although each participant was trained on how to insert the earplug into each ear, they were only fitted with a single earplug and the allocated ear was concealed from the researcher. This was achieved by asking each participant to choose a sealed envelope, half of which contained instructions to wear the earplug in the left ear and the remaining half contained instructions to 
wear the earplug in the right ear. The participant did not fit the earplug until leaving the test room on the first test session and they removed the earplug before entering the test room for each subsequent test session.

See Fig. 1 here

\subsection{Acoustic reflex thresholds}

Tympanometry was performed prior to measuring the ARTs. The equivalent ear canal volume $(\mathrm{ECV})$, an estimate of the volume of air trapped between the probe tip and the tympanic membrane (Fowler et al., 2002), was recorded to check this did not change during the study since this could affect the recorded value of the ART. The mean ECV at day 0 and 6 was $1.1 \mathrm{ml}( \pm 0.3)$ and $1.2 \mathrm{ml}( \pm 0.6)$ in the test ear and $1.1 \mathrm{ml}( \pm 0.3) 1.2 \mathrm{ml}( \pm 0.5)$ in the control ear, respectively. These changes are negligible and are unlikely to affect interpretation of the findings.

ARTs were measured on six occasions over an 8 day period: immediately before the use of the earplug (day 0), during earplug use (on day 2, 4 and 6) and after earplug use (4 hours and 24 hours). The ARTs were measured at these same times for the control ear. Ipsilateral and contralateral ARTs were measured using the GSI Tympstar middle ear analyzer with a 226 $\mathrm{Hz}$ probe tone. Ipsilateral measurements involved placing the measurement probe in the same ear as the reflex-eliciting stimulus. Contralateral measurements involved placing the measurement probe in the opposite ear from the reflex-eliciting stimulus. The stimuli used to elicit a reflex were pure tones at $0.5,1,2$ and $4 \mathrm{kHz}$. The order of the frequencies was 
counter-balanced between participants. Because the level of the ART eliciting stimulus may have exceeded the maximum output of the middle ear analyzer for some participants, we also used broadband (BBN), which can elicit a reflex at a lower sound level (Gefland, 1984). The stimuli were of fixed duration (1 second) and presented at an initial level of $70 \mathrm{~dB}$ HL (60 dB HL for BBN). The sound level was increased in $5 \mathrm{~dB}$ steps until the reflex was detected (reduction in compliance of $>0.02 \mathrm{~cm}^{3}$ ). Increasing the stimulus by a further $5 \mathrm{~dB}$ confirmed the reflex growth. The stimulus was decreased by $10 \mathrm{~dB}$ and increased in $2 \mathrm{~dB}$ steps to determine the ART. The stimulus was presented two additional times at the apparent ART to confirm repeatability and then increased by a further $2 \mathrm{~dB}$ to confirm reflex growth. If a change in compliance was not seen at the maximum stimulus eliciting level for a given frequency, $5 \mathrm{~dB}$ was added on the maximum value, following the procedure from previous earplug deprivation studies (Munro et al., 2009; Munro et al., 2014). Otoscopy was performed before tympanometry and ART measurements. The data included in the present study were taken from participants who did not show any evidence of pressure marks or cerumen impaction that may have occurred as a result of earplug use. The participants were also asked to take the earplug out immediately before entering the test room to ensure the investigator remained blinded to the plugged ear.

\subsection{Statistical analysis}

Statistical analysis consisted primarily of repeated-measures analysis of variance (ANOVA) using SPSS version 20. Post-hoc analysis included paired $t$-tests. The degrees of freedom were modified using the Greenhouse-Geisser correction when there was a statistically significant deviation from sphericity on Mauchly’s test (Kinnea et al,. 2009).

244 3. Results 
We investigated the effects of 6 days of unilateral auditory deprivation on ARTs. 24 participants completed the study and were included in the analysis. The time course of changes in the ipsilateral ARTs during the 6 days of wearing the earplug, as well as 4 and 24 hours after removing the earplug, are shown in Fig. 2. At baseline, the mean asymmetry in ARTs between the two ears was $<2 \mathrm{~dB}$ and was not statistically significant on paired $t$-tests. In the treatment ear, ARTs decreased over the 6 days (Fig. 2, top and middle row, filled symbols), and there was a slight, albeit much less pronounced increase of ARTs in the control ear (Fig. 2, top and middle row, open symbols), leading to an overall asymmetry of the ARTs between the ears (Fig. 2, bottom row). After removal of the earplug, ARTs started to recover towards baseline values.

\subsection{The time course for the onset and offset of changes in ARTs}

To characterize the time-course of changes in ARTs through unilateral conductive hearing loss by means of an earplug, we measured ipsilateral ARTs on days 2, 4 and 6 of earplug use (Fig. 2, top row). In the treatment ear, changes reached a maximum on day 4 or 6 , with a mean decrease of $4-5 \mathrm{~dB}$ for 2 and $4 \mathrm{kHz}$ and $\mathrm{BBN}$. In the control ear, changes were less pronounced, with increases in ARTs of 1-2 dB, and the magnitude of the effect was approximately comparable on all three test days. The raw data were analyzed for each reflexeliciting stimulus $(0.5,1,2$, and $4 \mathrm{kHz}$ pure tones and $\mathrm{BBN})$ using a two-factor (ear [2] $\mathrm{x}$ test 
session [4]) repeated-measures ANOVA. There was a significant effect of ear $(0.5 \mathrm{kHz}$,

$F(1.0,23.0)=11.45 ; p=0.003 ; 1 \mathrm{kHz}, F(1.0,23.0)=14.33 ; p=0.001 ; 2 \mathrm{kHz}, \quad F(1.0,23.0)$

$=15.17 ; p=0.001 ; 4 \mathrm{kHz}, \quad F(1.0,23.0)=9.95 ; p=0.004 ; \mathrm{BBN}, F(1.0,23.0)=22.91 ; p<$

0.001). There was also a significant interaction between ear and test session for the $2 \mathrm{kHz}, 4$

$\mathrm{kHz}$ and $\mathrm{BBN}$ stimuli $(F(3.0,69.0)=10.32 ; p<0.001 ; F(3.0,69.0)=4.42 ; p=0.007 F(2.0$, $46.4)=3.84 ; p=0.028$, respectively) indicating that the changes over time were different for each ear.

Next, we considered each ear independently using a one-factor (test session [4]) repeatedmeasures ANOVA at the three frequencies ( 2 and $4 \mathrm{kHz}$ and $\mathrm{BBN}$ ) that showed a significant interaction in the previous analysis. For all three stimuli ( 2 and $4 \mathrm{kHz}$ and $\mathrm{BBN}$ ) there was a significant effect of test session in the treatment ear $(F(2.2,50.8)=9.85 ; p<0.001 ; F(2.0$, $47.1)=6.28 ; p=0.004 ; F(2.0,45.1)=3.32 ; p=0.046$, respectively). There were no significant findings for the control ear.

Next, differences between the mean ARTs in the treatment ear at the different test sessions were analyzed using paired $t$-tests for each frequency individually, with a Bonferroni correction (with a significance level of $\alpha=0.05 / 6$ ) applied to account for multiple paired comparisons. For the $2 \mathrm{kHz}$ stimulus, there were significant differences between day 0 and day $4(p<0.001)$ and between day 0 and day $6(p<0.001)$. For the $4-\mathrm{kHz}$ stimulus, there were significant differences between day 0 and day $4(p=0.004)$ and between day 0 and 6 ( $p$ $=0.003)$. For the BBN stimulus, there were significant differences between day 0 and $4(p<$ 0.001). There were no significant differences between day 0 and $6(p=0.115)$. All other differences in mean ARTs between test days during earplug usage were not significant. 
Based on the findings from Kei (2012), the test-retest variability in ART (successive testing with the probe removed and reinserted) is $\leq 1 \mathrm{~dB}$ in all participants. Therefore, a change in ART of $>1 \mathrm{~dB}$ was used as a criterion change in ART in individual participants following unilateral earplug use. At $2 \mathrm{kHz}, 95 \%$ of the participants displayed a change of $>1 \mathrm{~dB}$ by day 2. At $4 \mathrm{kHz}, 71 \%$ participants exceeded $>1 \mathrm{~dB}$ by day 2 . Less participants exceeded the $>1$ $\mathrm{dB}$ criterion because for 8 participants, the ART exceeded the maximum stimulus eliciting level, preventing a larger change in ART from being measured.

We took the opportunity to analyze whether there was a correlation between earplug attenuation and the change in ART at $2 \mathrm{kHz}$ and $4 \mathrm{kHz}$ on day 4 and 6 of earplug use. Normality tests revealed that the data were not linear. Therefore, we carried out a Spearman's Rank Order Correlation. There were no significant correlations. asymmetry in ART between ears so that any change due to either ear was included. $115.0)=6.851, p<0.001 ; F(5.0,115.0)=3.650, p=0.004 ; F(3.08,71.0)=3.684, p=0.015$, respectively). However, the significant finding for BBN did not survive Bonferroni correction

\subsubsection{Recovery after earplug removal}

The recovery of ipsilateral ARTs was measured 4 and 24 hours after earplug removal. A clear trend of recovery to baseline levels was evident, with the biggest change occurring in the first 4 hours (Fig. 2). Although the change in the control ear was negligible, we analyzed the The difference in mean ear asymmetry (Fig 2, bottom panel) between all the time points was analyzed using a one-way (time [6]) repeated-measures ANOVA for each frequency separately. There was a significant effect for the $2 \mathrm{kHz}, 4 \mathrm{kHz}$ and $\mathrm{BBN}$ stimuli $(F(5.0$, $(\alpha=0.05 / 5)$. Next, the asymmetry in ipsilateral ARTs between the ears was analyzed using 
320 paired $t$-tests with a Bonferroni correction applied $(\alpha=0.05 / 16)$. At $2 \mathrm{kHz}$, there was a 321 significant difference between 4 hours and day $0(t(23)=-4.914, p<0.001)$ that survived 322 Bonferroni correction. There was also a significant difference, uncorrected, between 24 hours and day 0,24 hours and day 4, and 24 hours and day $6(t(23)=-2.331, p=0.029 ; t(23)=$ 2.953, $p=0.007 ; t(23)=2.050, p=0.052$, respectively). However, these did not survive Bonferroni correction. At $4 \mathrm{kHz}$, there was a statistically significant difference, uncorrected, between 4 hours and day 6, 24 hours and day 4, 24 hours and day $6(t(23)=2.452, p=0.022$; $t(23)=2.181, p=0.040 ; t(23)=2.963, p=0.007$, respectively). However, these did not survive Bonferroni correction (or the less conservative Turkey test).

\subsection{Frequency specificity of changes in ARTs}

Another aim of the study was to assess the frequency specificity of changes in ipsilateral

ART through auditory deprivation by means of an earplug. Mean changes in ipsilateral ARTs relative to baseline for the treatment and the control ear, are shown in Fig. 3. In the treatment ear, decreases in ARTs were more pronounced at the high frequencies ( 2 and $4 \mathrm{kHz}$; Fig. 3 , top panel).

Insert Fig 3 here

In the baseline condition (day 0), the mean absolute ART values at $4 \mathrm{kHz}$ were higher than at the other frequencies (Fig. 2). Statistical analysis was therefore carried out on the change in mean ARTs relative to baseline (Fig. 3), to avoid a significant finding due to a difference in absolute ART values between frequencies. A three factor (ear [2] x frequency [4] x test 
session [3] $)$ repeated-measures ANOVA revealed an effect of ear $(F(1.0,23.0)=10.99 ; p=$ $0.003)$ and a significant interaction between ear and frequency $(F(3.0,69.0=3.85 ; p=$ 0.013). Next, we considered each ear separately using a two-factor (frequency [4] $\mathrm{x}$ test session [3]) repeated-measures ANOVA. There was a significant effect of frequency in the treatment ear $(F(2.3,53.8)=6.07 ; p=0.003)$, but there was no significant interaction.

The change in mean ARTs in the treatment ear, collapsed over day 2, 4 and 6, was analyzed using paired $t$-tests with a Bonferroni correction applied for multiple paired comparisons $(\alpha=$ $0.05 / 6)$ of the four frequencies. $2 \mathrm{kHz}$ was significantly different from $0.5 \mathrm{kHz}(p=0.008)$ and $1 \mathrm{kHz}(p=0.006)$. Before a Bonferroni correction was applied, $4 \mathrm{kHz}$ was also significantly different from $0.5 \mathrm{kHz}(p=0.013)$ and $1 \mathrm{kHz}(p=0.017)$. The mean changes in ARTs in the control ear were small, and differences across frequencies were not significant.

The mean difference between the attenuation values between each frequency (including 2 $\mathrm{kHz}$ ) were analyzed using paired $t$-tests. There were significant differences between 0.5 and $1,0.5$ and 2 , and 0.5 and $4 \mathrm{kHz}(t(23.0)=10.91, p<0.001 ; t(23)=13.97, p<0.001 ; t(23)=$ 9.43, $p<0.001$, respectively), and between $1 \mathrm{kHz}$ and $2,1 \mathrm{~Hz}$ and $4, \mathrm{kHz}(t(23)=8.34, p$ $<0.001 ; t(23)=5.47, p<0.001$, respectively), which survived after Bonferroni correction (0.05/36). This suggests that the level of attenuation was significantly different between the low $(0.5$ and $1 \mathrm{kHz})$ and high frequencies $(2$ and $4 \mathrm{kHz})$, with the latter receiving the greatest level of attenuation from the earplug. Therefore, the absence of a significant effect between 4 and $0.5 \mathrm{kHz}$, and 4 and $1 \mathrm{kHz}$ on the ART measurement, cannot be attributed to an absence of a statistical difference between these frequencies on the attenuation values.

\subsection{Changes in ipsilateral versus contralateral ARTs}


370 All previous analyses in the present study investigated the ipsilateral ART. Next, the mean changes in ipsi- and contralateral ARTs relative to baseline were investigated (Fig. 4). For both the treatment and the control ear, ARTs measured in the ipsilateral as well as the contralateral ear showed similar trends, with decreases in ARTs when the ART was elicited by stimulating the treatment ear (Fig. 4, top row), and ARTs generally showing only little change from baseline when the ART was elicited by stimulating the control ear (Fig. 4, bottom row).

Insert Fig 4 here

We first investigated the change in mean ARTs for the ipsilateral and contralateral conditions, for presentation of the eliciting stimuli to the treatment ear (Fig 4, top row), relative to baseline (day 0 ). The measurement ear was the treatment ear for the ipsilateral condition and the control ear for contralateral condition and was denoted by the within-factor 'measurement ear'. The data were analyzed at each frequency using a two-factor (test session [3] x measurement ear [2]) repeated-measures ANOVA. There was a significant effect of test session for the $4 \mathrm{kHz}$ and $\mathrm{BBN}$ stimuli $(F(2.0,46.0)=4.806 ; p=0.013 ; F(2.0,46.0)=4.595$; $p=0.015$, respectively) but not measurement ear. However, these did not survive after a Bonferroni correction (with a significance level of $\alpha=0.05 / 5$ ).

Next, we investigated the change in mean ARTs for the ipsilateral and contralateral conditions, when the ARTs were measured in the treatment ear (Fig. 4, solid line in top and bottom row) relative to baseline (day 0). The presentation of the eliciting stimulus was the 
treatment ear for the ipsilateral condition and the control ear for the contralateral condition and was denoted by the within-factor 'stimulus ear'. The data were analyzed at each

397 frequency using a two-factor (test session [3] x stimulus ear [2]) repeated measures ANOVA.

398 There was a significant effect of stimulus ear for the $2 \mathrm{kHz}, 4 \mathrm{kHz}$ and BBN stimuli (F(1.0, $39923.0)=13.589 ; p=0.001 ; F(1.0,23.0)=34.193 ; p<0.001 ; F(1.0,23.0)=9.160 ; p=0.006$, 400 respectively). This means that the effect was different depending on stimulus ear, regardless 401 of time. For the $4 \mathrm{kHz}$ stimulus, there was also a significant interaction $(F(2.0,46.0)=6.311$; $p=0.004)$, which means that over time, the change in mean ART was different depending on 403 the stimulus ear.

404

405 In summary, the effect was significantly different when the ipsilateral and contralateral ARTs 406 were measured in the treatment ear. In contrast, there was an overall trend for the ipsilateral 407 and contralateral ARTs to be similar when the stimulus was presented to the treatment ear.

408

409 


\section{DISCUSSION}

411 The present study aimed to extend the work of Munro et al. (2009), Maslin et al. (2013) ad 412 Munro et al. (2014) by investigating: (1) the time course of changes in ARTs following 413 auditory deprivation; (2) the changes in the ART for a range of frequencies, and (3) the 414 location of change along the auditory pathway. The asymmetry between the ARTs in the two 415 ears immediately after termination of the monaural earplug treatment was primarily due to a 416 reduction in ART in the treatment ear of $4-5 \mathrm{~dB}$ from day 4 onwards for $2,4 \mathrm{kHz}$ and $\mathrm{BBN}$. 417 Recovery was evident by 4 and 24 hours after earplug removal at most frequencies. The 418 change in ART was primarily a high frequency effect and the same effect was observed in 419 different ears, when stimulating the treatment ear. Data were collected by a researcher 420 blinded to the treatment ear, and there were no changes in mean equivalent ear-canal volume across test session. Therefore, experimenter bias and differences in total-admittance probeinsertion depth can be ruled out as explanations for the changes in ART. The results offer evidence of frequency-specific sub-cortical plasticity following short-term unilateral auditory deprivation.

\subsection{The time course in the onset and offset of change}

\subsubsection{Onset of change}

In our study, changes in ARTs in the treatment ear reached significance from day 4 onwards. The onset of change in ARTs is similar to changes in spontaneous firing rates in the dorsal cochlear nucleus that have been reported in animal studies. In the study by Kaltenbach et al. (2000), the mean rate of spontaneous activity increased sharply from below normal levels on day 2 to levels that were significantly higher than normal on day 5 after unilateral tone exposure. The decrease at day 2 is likely to reflect an excitotoxically induced loss of neurons due to acoustic overstimulation during noise-induced hearing loss. As changes in spontaneous 
activity are related to changes in stimulus-evoked activity (Schaette and Kempter, 2006; 2009) we would therefore not expect to observe an increase in ART after 2 days of earplug use. Increases in spontaneous activity, as observed in the dorsal cochlear nucleus (DCN; Kaltenbach et al., 2000) and VCN (Vogler et al., 2011) have been implicated as a neural correlate of tinnitus (Kaltenbach et al., 2004; Koehler et al., 2013). Since the majority of human subjects report tinnitus during earplug-induced unilateral auditory (Schaette et al., 2012b), it is tempting to speculate about a common mechanism causing changes in ARTs and tinnitus. A candidate mechanism could be an increase in neuronal gain through homeostatic plasticity after hearing loss, which has been implicated to play a role in tinnitus development (Schaette et al., 2006; 2008; 2009).

The time course of changes in ARTs observed in the present study is consistent with homeostatic plasticity, a mechanism which acts to stabilize the mean neuronal activity over a time scale of hours to days (Turrigiano, 1999). In response to persistent reductions in neuronal activity, homeostatic plasticity scales up the strength of excitatory synapses, whereas inhibitory synapses are scaled down (Kilman et al., 2002; Turrigiano et al., 1998). Similar changes have been observed in an animal model after an earplug period of 24 hours (Whiting et al., 2009). An earplug does not, of course, result in overstimulation of the auditory system, which can be a consequence of noise induced hearing loss, leading to an excitotoxically induced loss of neurons (Kaltenbach et al., 2000). The initial reduction in neural activity reported by Kaltenbach et al. (2000) is therefore not observed following earplug use (Whiting et al., 2009).

A reduction of inhibition in conjunction with an increase in excitation would lead to an increase in neural gain, which could cause a reduction in the ART (Maslin et al., 2013; 
460 Munro et al., 2009; Munro et al., 2014). The present study was able to demonstrate a trend of 461 reducing ART after 2 days of unilateral earplug use. However, measurements were not made 462 prior to 2 days. Therefore, based on Whiting et al. (2009), it is possible that an even shorter 463 duration would reveal a trend of changing neural gain.

The interpretation that the findings from the present study may reflect an increase in excitation and a reduction in inhibition is in contrast to the findings of Popescu et al. (2010). However, the results of Popescu et al. (2010) may not be comparable to the present finding since the recordings were made under pentobarbital sodium anesthesia and this has been shown to decrease the magnitude of evoked responses in the SOC. There is extensive animal literature suggesting that neural gain increases after auditory deprivation (Kaltenbach et al., 2000; Mulders et al., 2009; Norena et al., 2003). However, caution should be applied to direct comparisons between studies due to differences in methodology, species, time of measurements etc. For example, much of the animal research used noise exposure to induce a hearing loss (Kaltenbach et al., 2000; Mulders et al., 2009; Norena et al., 2003), which inflicts trauma and hair cells loss (Kujawa et al., 2009). Such damage does not occur during earplug use.

\subsubsection{Offset of change}

Compared to baseline, ear asymmetry at $2 \mathrm{kHz}$ was significantly larger 4 hours but not 24 hours after earplug removal. In other words, the effect disappeared by 4-24 hours at most frequencies affected by the earplug. This is, to the authors' knowledge, the first study to demonstrate a trend of recovery in ARTs towards baseline level as early as 4 hours after earplug removal. 
Munro et al. (2014) reported that most of the difference between the ears had disappeared within 24 hours after the removal of the earplug. A change in excitatory and inhibitory synapse strength reversing within 24 hours has also been observed after the removal of the earplug in adult rats (Whiting et al., 2009). It is possible that the acoustic environment influences the recovery of ART after earplug removal. This was not controlled for in the present study or in the previous ART study by Munro et al. (2014). In our study, the first measurement after earplug removal was carried out after 4 hours, and the participants (students) may have stayed on-site in acoustically quiet environments such as a library during this time. In the study by Munro et al. (2014), on the other hand, participants were only tested 24 hours after the removal of the earplug, and might have spent this time period in a normal, louder acoustic environment. Therefore, there might have been relevant differences in the acoustic stimulation during recovery in the two studies that were not controlled for, which could explain the (slight) differences in outcomes. A useful future study could control for the acoustic environment of the recovery period and could also investigate if adaptation to 'quiet' or 'loud' acoustic environments operates on different time scales.

Another observation that can be made from the present study is that the onset of changes in ARTs following earplug use was slower than the offset of changes after removal of the earplug: the asymmetry between the ears at day 2 of earplug use was similar to the asymmetry between the ears observed 4 hours after earplug removal. These trends raise intriguing questions about the mechanism behind the onset and offset of change and warrant further investigation. Other mechanisms of neuronal adaptation have also been shown to have different time constants for on- and offset. It has, for example, been shown that adaptive coding in the inferior colliculus of guinea pigs, a mechanism which shifts neuronal response functions in response to changes in the acoustic environment within hundreds of 
milliseconds, reacts significantly faster to an increase in sound intensity than to a decrease

511 (Dean et al., 2008). However, this mechanism operates on a much faster time scale than

512 homeostatic plasticity (Turrigiano et al., 1998). Homeostatic plasticity is inert to such fast

513 changes in the environment, which can activate other plasticity mechanisms operating on a

514 shorter time scale that are not involved in maintaining neural stability, but instead alter synapses in a specific way to store information (Zenke et al., 2013). Dean et al. (2008) described a mechanism that has a functional role of ensuring coding efficiency over a wide range of sound levels, by shifting the position of the neural dynamic range in response to changing sound level statistics in the acoustic environment (Dean et al., 2008).

520 Homeostatic plasticity involves synaptic scaling which, as mentioned previously, has been demonstrated to be a relatively slow process (Turrigiano, 1999). Under some circumstances synaptic scaling may occur within 1 hour (Ibata et al., 2008). However, this rapid time scale of change was related to synaptic upscaling (onset), not synaptic downscaling (offset). Regardless, evidence of homeostatic plasticity operating on a time scale of 1 hour could still offer an explanation for the more rapid offset of change in ART, as demonstrated in the present study. Therefore, further research is required to understand which auditory characteristics, e.g. sound level or nature of the sound, in the acoustic environment determine how quickly homeostatic plasticity operates. It is conceivable that transition to a louder acoustic environment (i.e. taking the earplug out) could result in a faster change. Following on from this, a further study with more focus on directly comparing the time course of the onset and offset of changes in ARTs is therefore suggested. 
534 The earplugs used to create auditory deprivation in our study attenuated high frequencies more strongly than low frequencies (Fig. 1). The ART measurements showed a significant effect of frequency for ipsilateral ARTs in the treatment ear, where we observed smaller changes at lower frequencies $(0.5$ and $1 \mathrm{kHz})$ and larger changes at higher frequencies $(2$ and $4 \mathrm{kHz}$ ) (Fig. 3). This finding suggests that the changes in ARTs are indeed manifestations of a frequency-specific plasticity response. This conclusion is further supported by the finding of large changes in ARTs for BBN (Fig. 2) which comprises the frequency range where the earplug had maximum effect. However, only the changes in ARTs at $2 \mathrm{kHz}$ were significantly different from those at the lower frequencies. Differences between changes at 4 $\mathrm{kHz}$ and 0.5 or $1 \mathrm{kHz}$ just failed to achieve significance after a Bonferroni correction for multiple paired comparisons. The significant finding at $2 \mathrm{kHz}$ and not $4 \mathrm{kHz}$ could be explained by the basalward shift in the travelling wave: at high sound levels, pure tones maximally excite the region of the cochlea with a characteristic frequency (the frequency of a sound at which the threshold of the auditory nerve is lowest) half an octave above the tone frequency (Plack, 2013). Therefore, the significant difference in the mean change in ART in the treatment ear at $2 \mathrm{kHz}$ compared to $0.5 \mathrm{kHz}$ and $1 \mathrm{kHz}$ could reflect a contribution from the $3 \mathrm{kHz}$ region of the basilar membrane, where the earplug provided maximum attenuation (Fig. 1). Unfortunately, the test equipment did not allow direct measurements at $3 \mathrm{kHz}$. However, a significant effect at $1 \mathrm{kHz}$ should have also been expected to occur, if there was a contribution from the $2 \mathrm{kHz}$ region. Instead, the non-significant effect at $4 \mathrm{kHz}$ could reflect high variability and lack of power.

Nevertheless, a significant change in mean ART at $2 \mathrm{kHz}$ compared to 0.5 and $1 \mathrm{kHz}$ is still evidence of a frequency-specific change in neural gain. This finding is consistent with the predictions of the computational model by Schaette et al. (2006), where activity stabilization 

in the auditory system that is proportional to the corresponding hearing threshold loss.

561

562

through homeostatic plasticity after hearing loss causes a frequency-specific increase in gain

However, the frequency effect differs depending on what outcome measure is being used. For example, the change in loudness after unilateral auditory deprivation was observed in both ears and over a wide range of frequencies (Formby et al., 2003; 2007). This is distinct to the ART findings in the present study, in Munro and Blount (2009) and in Munro et al. (2014). It is possible that there are two distinct neural gain control mechanisms underlying the change in ART and loudness. At the present time, it is not possible to identify a specific location in the auditory pathway at which there is a change in neural gain. If this mechanism is distinct from the acoustic reflex gain control mechanism, one can hypothesize that the neural gain control mechanism for loudness operates above the level of the acoustic reflex arc. However, the change in loudness may simply represent a change in the behavioral response criterion of the participant. For example, when the earplug is removed, sounds may be judged as being louder than before the period of deprivation. This alternative interpretation is supported by evidence of a reduction in loudness discomfort levels in factory workers following retirements (Niemeyer, 1971).

The frequency-specificity of such plasticity mechanisms in the auditory system could be investigated in more detail in a future study with active earplugs providing specifically shaped patterns of attenuation, or with hearing aids with different frequency bands amplified. Furthermore, using measurement procedures that are not limited to high sound levels (e.g. investigating the input-output function of the ABR) will eliminate any contribution from the upward spread of excitation on the basilar membrane on the results. 


\subsection{Changes in ipsilateral versus contralateral ARTs}

585 The present study was able to demonstrate a reduction in the ART following earplug use when the stimulus was presented to the treatment ear, regardless of which ear the reflex was being measured (Fig. 4). In contrast, there was a significant difference in the mean ART after earplug use when comparing measurements when the stimulus was presented to the control ear, regardless of the ear of measurement. As the change in ipsilateral ART in the treatment ear was not observed when the stimulus is presented to the control ear in the contralateral measurement, these findings offer evidence that the change in neural gain is unlikely to operate in the descending limb of the acoustic reflex arc (Lee et al., 2006). The findings are therefore likely to represent a change in neural gain in the ascending limb of the acoustic reflex arc, which would be consistent with a similar magnitude of change in ART in the ipsilateral and contralateral measurement when the stimulus was presented to the treatment ear.

The VCN is the first auditory nucleus in the acoustic reflex arc. Therefore, a change in the cochlear nucleus in the present study would be consistent with reports of increased spontaneous and stimulus-evoked activity in the cochlear nucleus following acoustic trauma (Cai et al., 2009; Kaltenbach et al., 2000; Vogler et al., 2011). This finding would also be consistent with studies modeling the neural gain mechanism (Schaette et al., 2006). However, the findings in the present study do not eliminate the possibility of a change in neural gain first occurring at a higher level in the ascending acoustic reflex arc, e.g. superior olivary complex. Further work using measures such as the ABR needs to be done to establish where along the ascending auditory pathway the change in neural gain is occurring. Furthermore, to 607 confidently eliminate the possibility of a top-down influence via the descending medial 
608 olivocochlear complex pathway accounting for the change in ART, a future study could incorporate a measure of MOC activity such as otoacoustic emissions.

611 The majority of participants reported informally the presence of phantom auditory sensations 612 during earplug use in the current study. Phantom auditory sensations have been shown to be 613 induced in normal hearing listeners after a short period of unilateral earplug use (Schaette et 614 al., 2012). Tinnitus is a phantom auditory sensation often associated with a hearing loss 615 (Axelsson et al., 1989). This suggests that the mechanism responsible for changes in ART following earplug deprivation could be similar for some reports of tinnitus in a clinical 617 population (Schaette and Kempter, 2006; 2009). The time course of recovery of ART back to 618 baseline levels in the present study is similar to Schaette et al. (2012) who reported that the phantom sounds disappeared immediately after the removal of the earplug, with only four participants still reporting phantom sounds at the end of the day. A future study investigating a change in ART after earplug use could incorporate a similar outcome measure of phantom sounds used by Schaette et al. (2012). If a change in ART and an emergence of phantom sounds is reported, this would support the hypothesis that the same gain mechanism is involved in the acoustic reflex and phantom auditory perceptions, i.e., tinnitus.

If the physiological adaptive mechanisms underlying tinnitus and hyperacusis are the same as the mechanisms responsible for the changes in ART, then the findings from the present study could be clinically relevant (Brotherton et al., 2015). For example, a significant change in the treatment ear after 4 days of unilateral earplug use suggests that 4 days may be needed for a sound device treatment to effectively reduce the enhanced neural gain in tinnitus and hyperacusis. However, the present study did not investigate a clinical intervention and further research is required to confirm if this is the case. If the neural gain mechanism underlying the 
633 change in ART after earplug use is frequency specific, this may offer an explanation for

634 reports that an increase in neural gain predicted from the audiograms of individuals with

635 hearing loss is consistent with the pitch of tinnitus perceived by these individuals (Schaette et

636 al., 2009). However, a frequency specific effect has not been reported in loudness judgments

637 after earplug use (Formby et al., 2003; 2007; Munro et al, 2014). Although this is consistent

638 with reports of hyperacusis generally showing a change in loudness judgments across a range

639 of frequencies (Anari et al., 1999; Sheldrake et al., 2015), it cannot account for abnormal

640 loudness in a tinnitus cohort only at frequencies outside the hearing loss region (Hebert et al.,

641 2013). An alternative explanation for the development of hyperacusis comes from reports that

642 type II cochlear afferents may not be involved in the acoustic reflex arc (Maison et al., 2016).

643 Instead, type II cochlear afferents could act as a pain pathway (Flores et al., 2015; Liu et al., 644 2015), which at low sound levels could evoke erroneous activity leading to a painful 645 hypersensitivity to sounds. A final point is in regard to ART as an outcome measure. For 646 tinnitus research, using the ART as an outcome measure may not be appropriate. Fernandes et 647 al. (2013) has reported that contralateral reflexes are elevated in tinnitus patients. Therefore, 648 rather than the ART, it may be more suitable to use the ABR as an outcome measure in 649 tinnitus patients, as used by Schaette et al. (2011) and Gu et al. (2012).

650

\section{5. Conclusions}

652 This study is novel in showing that the asymmetry between the ARTs in the treatment and the control ear is evident from day 4 and at the frequencies that received the greatest attenuation.

654 Recovery was shown to occur 4 hours after the removal of the earplug at most frequencies.

655 The changes in ART were observed in both ears, when stimulating the treatment ear. The

656 findings can be explained by a homeostatic neural gain mechanism that operates in the ascending limb of the acoustic reflex arc. There is evidence to suggest that the onset of 
658 change during earplug use is slower than the offset of change following removal of the 659 earplug. However, a clearer understanding of the time course of change is required. A better 660 understanding of the neural gain mechanism could contribute to the development of sound 661 treatments for tinnitus and hyperacusis. Evidence of a neural gain control mechanism has 662 been shown in other sensory system (Merabet et al., 2004; Rossini et al., 1994; Wu et al., 663 2012); therefore the findings from the present study, could be applicable to other sensory 664 systems.

665 
666 DECLARATION OF CONFLICTING INTEREST

667 The Authors declare that there is no conflict of interest

668 
669 SUMBISSION DECLARATION

670 All the authors have approved the final article

671 


\section{HUMAN RIGHTS}

673 Informed consent was obtained for experimentation with human subjects

674 The privacy rights of human subjects was always observed

675

676

677 


\section{FUNDING}

679 This work was supported by the University of Manchester

680 


\section{References}

682

683

684

685

686

687

688

689

690

691

692

693

694

695

696

697

698

699

700

701

702

703

704

705

706

707

708

709

710

711

712

713

714

715

716

717

718

Anari, M., Axelsson, A., Eliasson, A., Magnusson, L. 1999. Hypersensitivity to sound--questionnaire data, audiometry and classification. Scand Audiol 28, 219-30.

Andersson, G., Lindvall, N., Hursti, T., Carlbring, P. 2002. Hypersensitivity to sound (hyperacusis): a prevalence study conducted via the internet and post. Int J Audiol 41, 545-554.

Auerbach, B.D., Rodrigues, P.V., Salvi, R.J. 2014. Central gain control in tinnitus and hyperacusis. Front Neurol 5, 206.

Axelsson, A., Ringdahl, A. 1989. Tinnitus--a study of its prevalence and characteristics. Br. J. Audiol. 23, 53-62.

Brotherton, H., Plack, C.J., Maslin, M., Schaette, R., Munro, K.J. 2015. Pump Up the Volume: Could Excessive Neural Gain Explain Tinnitus and Hyperacusis? Audiol. Neurootol. 20, 273-282.

Cai, S.Q., Ma, W.L.D., Young, E.D. 2009. Encoding Intensity in Ventral Cochlear Nucleus Following Acoustic Trauma: Implications for Loudness Recruitment. J Assoc Res Otolaryngol 10, 5-22.

Campo, P., Maguin, K., Lataye, R. 2007. Effects of aromatic solvents on acoustic reflexes mediated by central auditory pathways. Toxicol. Sci. 99, 582-590.

Dawes, P., Fortnum, H., Moore, D.R., Emsley, R., Norman, P., Cruickshanks, K., Davis, A., EdmondsonJones, M., McCormack, A., Lutman, M., Munro, K. 2014. Hearing in Middle Age: A Population Snapshot of 40-to69-Year Olds in the United Kingdom. Ear Hear. 35, E44-E51.

Dean, I., Robinson, B.L., Harper, N.S., McAlpine, D. 2008. Rapid neural adaptation to sound level statistics. J Neurosci 28, 6430-8.

Decker, T.N., Howe, S.W. 1981. Short-Term Auditory Deprivation - Effect on Brain-Stem Electrical Response. Hear. Res. 4, 251-263.

Eggermont, J.J., Roberts, L.E. 2014. Tinnitus: animal models and findings in humans, Cell Tissue Res., 2014/10/01 ed.

Fernandes, L.D., Momensohn-Santos, T.M., Carvalho, J.S.M., Carvalho, F.L.D. 2013. Tinnitus and Normal Hearing: A Study on Contralateral Acoustic Reflex. Am J Audiol 22, 291-296.

Flores, E.N., Duggan, A., Madathany, T., Hogan, A.K., Marquez, F.G., Kumar, G., Seal, R.P., Edwards, R.H., Liberman, M.C., Garcia-Anoveros, J. 2015. A Non-canonical Pathway from Cochlea to Brain Signals Tissue-Damaging Noise. Curr. Biol. 25, 606-612.

Formby, C., Sherlock, L.P., Gold, S.L. 2003. Adaptive plasticity of loudness induced by chronic attenuation and enhancement of the acoustic background. J. Acoust. Soc. Am. 114, 55-58.

Formby, C., Sherlock, L.G.P., Gold, S.L., Hawley, M.L. 2007. Adaptive Recalibration of Chronic Auditory Gain. Semin Hear 28, 295-302.

Fowler, C.G., Shanks, J.E. 2002. Tympanometry. 5th ed ed. Lippincott Williams \& Wilkins, Balitmore.

Gefland, S.A. 1984. "The contralateral acoustic reflex," in The Acoustic Reflex: Basic Principles and Clinical Application Academic, Orlando, FL.

Gu, J.W., Herrmann, B.S., Levine, R.A., Melcher, J.R. 2012. Brainstem Auditory Evoked Potentials Suggest a Role for the Ventral Cochlear Nucleus in Tinnitus. J Assoc Res Otolaryngol 13, 819833.

Hebert, S., Fournier, P., Norena, A. 2013. The Auditory Sensitivity is Increased in Tinnitus Ears. J. Neurosci. 33, 2356-2364.

Ibata, K., Sun, Q., Turrigiano, G.G. 2008. Rapid synaptic scaling induced by changes in postsynaptic firing. Neuron 57, 819-826.

Kaltenbach, J.A., Zhang, J.S., Afman, C.E. 2000. Plasticity of spontaneous neural activity in the dorsal cochlear nucleus after intense sound exposure. Hear. Res. 147, 282-292.

Kaltenbach, J.A., Zacharek, M.A., Zhang, J., Frederick, S. 2004. Activity in the dorsal cochlear nucleus of hamsters previously tested for tinnitus following intense tone exposure. Neurosci Lett 355, 121-5.

Kei, J. 2012. Acoustic Stapedial Reflexes in Healthy Neonates: Normative Data and Test-Retest Reliability. J. Am. Acad. Audiol. 23, 46-56. 
Kilman, V., van Rossum, M.C., Turrigiano, G.G. 2002. Activity deprivation reduces miniature IPSC amplitude by decreasing the number of postsynaptic $G A B A(A)$ receptors clustered at neocortical synapses. J Neurosci 22, 1328-37.

Kinnea, P.R., Gray, C.D. 2009. SPSS 16 made simple Psychology Press, Hove, UK.

Koehler, S.D., Shore, S.E. 2013. Stimulus timing-dependent plasticity in dorsal cochlear nucleus is altered in tinnitus. J Neurosci 33, 19647-56.

König, O., Schaette, R., Kempter, R., Gross, M. 2006. Course of hearing loss and occurrence of tinnitus. Hear Res 221, 59-64.

Kujawa, S.G., Liberman, M.C. 2009. Adding Insult to Injury: Cochlear Nerve Degeneration after "Temporary" Noise-Induced Hearing Loss. J. Neurosci. 29, 14077-14085.

Lee, D.J., de Venecia, R.K., Guinan, J.J., Brown, M.C. 2006. Central auditory pathways mediating the rat middle ear muscle reflexes. Anat Rec Part A 288A, 358-369.

Liu, C., Glowatzki, E., Fuchs, P.A. 2015. Unmyelinated type II afferent neurons report cochlear damage. Proc. Natl. Acad. Sci. U. S. A. 112, 14723-14727.

Maison, S., Liberman, L., D., Liberman, M.C. 2016. type-II cochlear ganlgion neurons do not drive the olivocochlear reflex: re-examination of the cochlear phenotype in peripherin knockout mice. Society of Neuroscience.

Maslin, M.R.D., Munro, K.J., Lim, V.K., Purdy, S.C., Hall, D.A. 2013. Investigation of cortical and subcortical plasticity following short-term unilateral auditory deprivation in normal hearing adults. Neuroreport 24, 287-291.

Merabet, L.B., Maguire, D., Warde, A., Alterescu, K., Stickgold, R., Pascual-Leone, A. 2004. Visual hallucinations during prolonged blindfolding in sighted subjects. J. Neuroophthalmol. 24, 109-113.

Mulders, W.H.A.M., Robertson, D. 2009. Hyperactivity in the Auditory Midbrain after Acoustic Trauma: Dependence on Cochlear Activity. Neuroscience 164, 733-746.

Munro, K.J., Blount, J. 2009. Adaptive plasticity in brainstem of adult listeners following earpluginduced deprivation. J. Acoust. Soc. Am. 126, 568-571.

Munro, K.J., Merrett, J.F. 2013. Brainstem plasticity and modified loudness following short-term use of hearing aids. J. Acoust. Soc. Am. 133, 343-349.

Munro, K.J., Turtle, C., Schaette, R. 2014. Plasticity and modified loudness following short-term unilateral deprivation: Evidence of multiple gain mechanisms within the auditory system. J. Acoust. Soc. Am. 135, 315-322.

Niemeyer, W. 1971. Relations between Discomfort Level and Reflex Threshold of Middle Ear Muscles. Audiology 10, 172-176.

Norena, A.J., Eggermont, J.J. 2003. Changes in spontaneous neural activity immediately after an acoustic trauma: implications for neural correlates of tinnitus. Hear. Res. 183, 137-153.

Plack, C.J. 2013. The Sense of Hearing. 2 ed. Psychology Press, Hove.

Popescu, M.V., Polley, D.B. 2010. Monaural Deprivation Disrupts Development of Binaural Selectivity in Auditory Midbrain and Cortex. Neuron 65, 718-731.

Rossini, P.M., Martino, G., Narici, L., Pasquarelli, A., Peresson, M., Pizzella, V., Tecchio, F., Torrioli, G., Romani, G.L. 1994. Short-term brain 'plasticity' in humans: transient finger representation changes in sensory cortex somatotopy following ischemic anesthesia. Brain Res. 642, 169-77.

Schaette, R., Kempter, R. 2006. Development of tinnitus-related neuronal hyperactivity through homeostatic plasticity after hearing loss: a computational model. Eur. J. Neurosci. 23, 31243138.

Schaette, R., Kempter, R. 2008. Development of hyperactivity after hearing loss in a computational model of the dorsal cochlear nucleus depends on neuron response type. Hear. Res. 240, 5772.

Schaette, R., Kempter, R. 2009. Predicting Tinnitus Pitch From Patients' Audiograms With a Computational Model for the Development of Neuronal Hyperactivity. J. Neurophysiol. 101, 3042-3052. 
Schaette, R., McAlpine, D. 2011. Tinnitus with a Normal Audiogram: Physiological Evidence for Hidden Hearing Loss and Computational Model. J. Neurosci. 31, 13452-13457.

Schaette, R., Turtle, C., Munro, K.J. 2012. Reversible Induction of Phantom Auditory Sensations through Simulated Unilateral Hearing Loss, Plos One, Vol. 7.

Sereda, M., Hall, D.A., Bosnyak, D.J., Edmondson-Jones, M., Roberts, L.E., Adjamian, P., Palmer, A.R. 2011. Re-examining the relationship between audiometric profile and tinnitus pitch. Int J Audiol 50, 303-312.

Sheldrake, J., Diehl, P.U., Schaette, R. 2015. Audiometric characteristics of hyperacusis patients. Frontiers in Neurology 6.

Turrigiano, G.G. 1999. Homeostatic plasticity in neuronal networks: the more things change, the more they stay the same (vol 21, pg 221, 1998) (vol 22, pg 280, 1999). Trends Neurosci. 22, 416-416.

Turrigiano, G.G., Leslie, K.R., Desai, N.S., Rutherford, L.C., Nelson, S.B. 1998. Activity-dependent scaling of quantal amplitude in neocortical neurons. Nature 391, 892-896.

Vogler, D.P., Robertson, D., Mulders, W.H.A.M. 2011. Hyperactivity in the Ventral Cochlear Nucleus after Cochlear Trauma. J. Neurosci. 31, 6639-6645.

Whiting, B., Moiseff, A., Rubio, M.E. 2009. Cochlear Nucleus Neurons Redistribute Synaptic Ampa and Glycine Receptors in Response to Monaural Conductive Hearing Loss. Neuroscience 163, 1264-1276.

Wu, K.N., Tan, B.K., Howard, J.D., Conley, D.B., Gottfried, J.A. 2012. Olfactory input is critical for sustaining odor quality codes in human orbitofrontal cortex. Nat. Neurosci. 15, 1313-U189.

Zenke, F., Hennequin, G., Gerstner, W. 2013. Synaptic Plasticity in Neural Networks Needs Homeostasis with a Fast Rate Detector. Plos Comput Biol 9. 
Table I. Summary of studies investigating the ART following a period acute deprivation or augmented stimulation in normal hearing adults

\begin{tabular}{|c|c|c|c|}
\hline Author & Condition & Measure & Results \\
\hline Decker et al. (1981) & $\begin{array}{l}\text { Unilateral } \\
\text { earplug } \\
\text { deprivation }\end{array}$ & $\begin{array}{l}\text { Ipsilateral } 0.5,1 \& 2 \mathrm{kHz} \text { at } \\
\text { baseline, } 10,20 \text { and } 30 \text { hours }\end{array}$ & $\begin{array}{l}\text { A reduction of around } 3 \mathrm{~dB} \text { in the treatment ear } 10 \text { hours after earplug use. The } \\
\text { change in the control ear was variable across frequencies showing a decrease of } \\
2 \mathrm{~dB} \text { and an increase of } 1 \mathrm{~dB} 10 \text { hours after earplug. A similar change in ART } \\
\text { was observed } 20 \text { and } 30 \text { hours after earplug use. The change was statistically } \\
\text { significant only at } 2 \mathrm{kHz} \text {. }\end{array}$ \\
\hline Munro et al. (2009) & $\begin{array}{l}\text { Unilateral } \\
\text { earplug } \\
\text { deprivation }\end{array}$ & $\begin{array}{l}\text { Ipsilateral } 2 \& 4 \mathrm{kHz} \text { at baseline \& } \\
7 \text { days }\end{array}$ & $\begin{array}{l}\text { A significant reduction of around } 8 \mathrm{~dB} \text { in the treatment ear, and a significant } \\
\text { reduction of around } 3 \mathrm{~dB} \text { in the control ear after } 7 \text { days of unilateral earplug use. } \\
\text { A similar reduction was observed for } 2 \text { and } 4 \mathrm{kHz} \text {. }\end{array}$ \\
\hline Munro et al. (2013) & $\begin{array}{l}\text { Unilateral } \\
\text { hearing aid use }\end{array}$ & $\begin{array}{l}\text { Ipsilateral } 0.5,2 \mathrm{kHz} \& \mathrm{BBN} \text { at } \\
\text { baseline, } 3 \text { and } 5 \text { days }\end{array}$ & $\begin{array}{l}\text { An increase of around } 2 \mathrm{~dB} \text { in the treatment ear and a reduction of around } 2 \mathrm{~dB} \\
\text { in the control ear } 3 \text { days after earplug use. The difference in ART between the } \\
\text { ears was marginally significant difference between } 0.5 \text { and } 2 \mathrm{kHz} \text {. }\end{array}$ \\
\hline Maslin et al. (2013) & $\begin{array}{l}\text { Unilateral } \\
\text { earplug use }\end{array}$ & $\begin{array}{l}\text { Ipsilateral } 0.5 \& 4 \mathrm{kHz} \text { at baseline } \\
\text { and } 7 \text { days }\end{array}$ & $\begin{array}{l}\text { A reduction of around } 7 \mathrm{~dB} \text { in the treatment ear and an increase of around } 2 \mathrm{~dB} \\
\text { in the control ear after earplug use. The change in ART was larger at } 4 \mathrm{kHz} \\
\text { compared to } 0.5 \mathrm{kHz} \text {. This difference between frequencies was not statistically } \\
\text { significant. }\end{array}$ \\
\hline Munro et al. (2014) & $\begin{array}{l}\text { Unilateral } \\
\text { earplug use }\end{array}$ & $\begin{array}{l}\text { Ipsilateral } 0.5,2 \mathrm{kHz} \& \mathrm{BBN} \text { at } \\
\text { baseline and } 7 \text { days of earplug use, } \\
1 \text { and } 7 \text { days after earplug removal }\end{array}$ & $\begin{array}{l}\text { A reduction of around } 5 \mathrm{~dB} \text { in the treatment ear and an increase of around } 2 \mathrm{~dB} \\
\text { in the control ear after earplug use. The change in ART was larger at } 2 \mathrm{kHz} \\
\text { compared to } 0.5 \mathrm{kHz} \text {, but this difference between frequencies was not } \\
\text { statistically significant. Most of the asymmetry between the ears disappeared } \\
\text { within } 1 \text { day of earplug removal. }\end{array}$ \\
\hline
\end{tabular}


808 Fig. 1. Mean attenuation values taken on day 0 of earplug use for the first fitting (grey open circle with dotted line), second fitting (grey closed circle with solid line), third fitting (black open circle with dotted line) and the mean attenuation values averaged across the three fittings (black closed circle with solid line). Errors bars show \pm 1 standard deviation $(n=24)$. Fig. 2. Time course of changes in ARTs during 6 days of earplug use, and 4 and $24 \mathrm{~h}$ after removal of the earplug. ARTs were elicited with pure tones $(0.5,1,2$, or $4 \mathrm{kHz})$ or broadband noise (BBN). The top row shows the mean ARTs from the treatments ears (filled circles) and the control ears (open circles). In the middle row, changes from the pre-earplug baseline values at day 0 are shown for the control (open squares) and the plugged ears (filled squares). The bottom row shows the development of the asymmetry in ART between the ears (control - treatment) over time. The vertical dotted lines indicate the time point at which the earplug was removed (day 6$)$. Errors bars show \pm 1 standard deviation $(n=24)$.

Fig. 3. Frequency-specificity of earplug-induced changes in ARTs. a) Changes in ipsilateral ARTs from pre-earplug baseline in the treatment ear at day 2 (squares with dotted line), day 4 (diamonds with dashed line), and day 6 (circles with solid line). b) Changes in ipsilateral ARTs in the control ear, line styles as in (a). Errors bars show \pm 1 standard deviation $(n=24)$. Fig. 4. Changes in ipsi- and contralateral ARTs after auditory deprivation through an earplug. All graphs show changes from the pre-earplug baseline at day 0 . Solid lines denote measurements where the ART was measured ipsilateral to the presentation of the eliciting stimulus, dashed lines show results for contralateral ART measurements. The top row shows ART changes for presentation of the eliciting stimuli to the treatment ear, and the bottom row for presentation to the control ear. Errors bars show \pm 1 standard deviation $(n=24)$. 


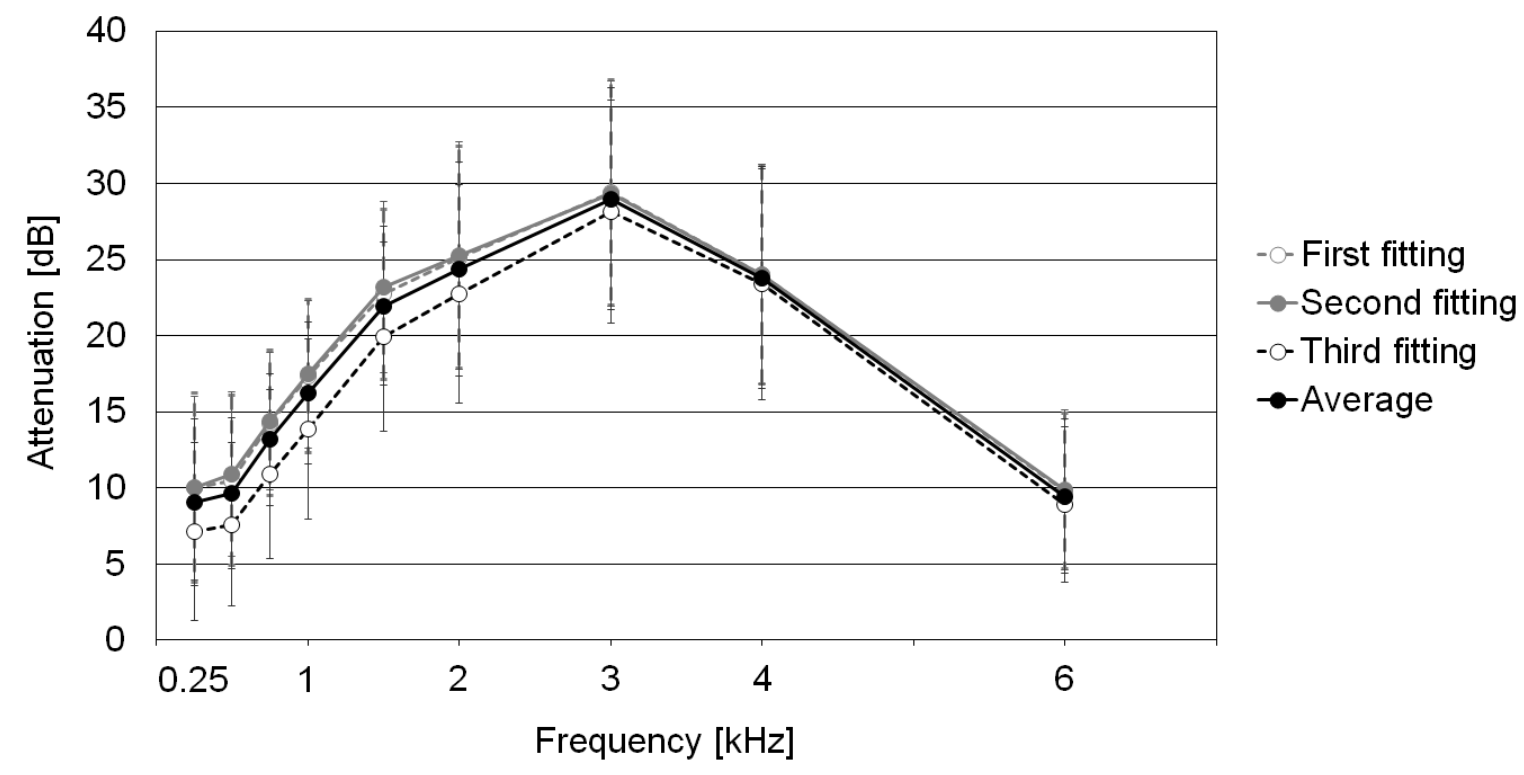

833 Fig. 1. Mean attenuation values taken on day 0 of earplug use for the first fitting (grey open

834 circle with dotted line), second fitting (grey closed circle with solid line), third fitting (black

835 open circle with dotted line) and the mean attenuation values averaged across the three 836 fittings (black closed circle with solid line). Errors bars show \pm 1 standard deviation $(n=24)$. 


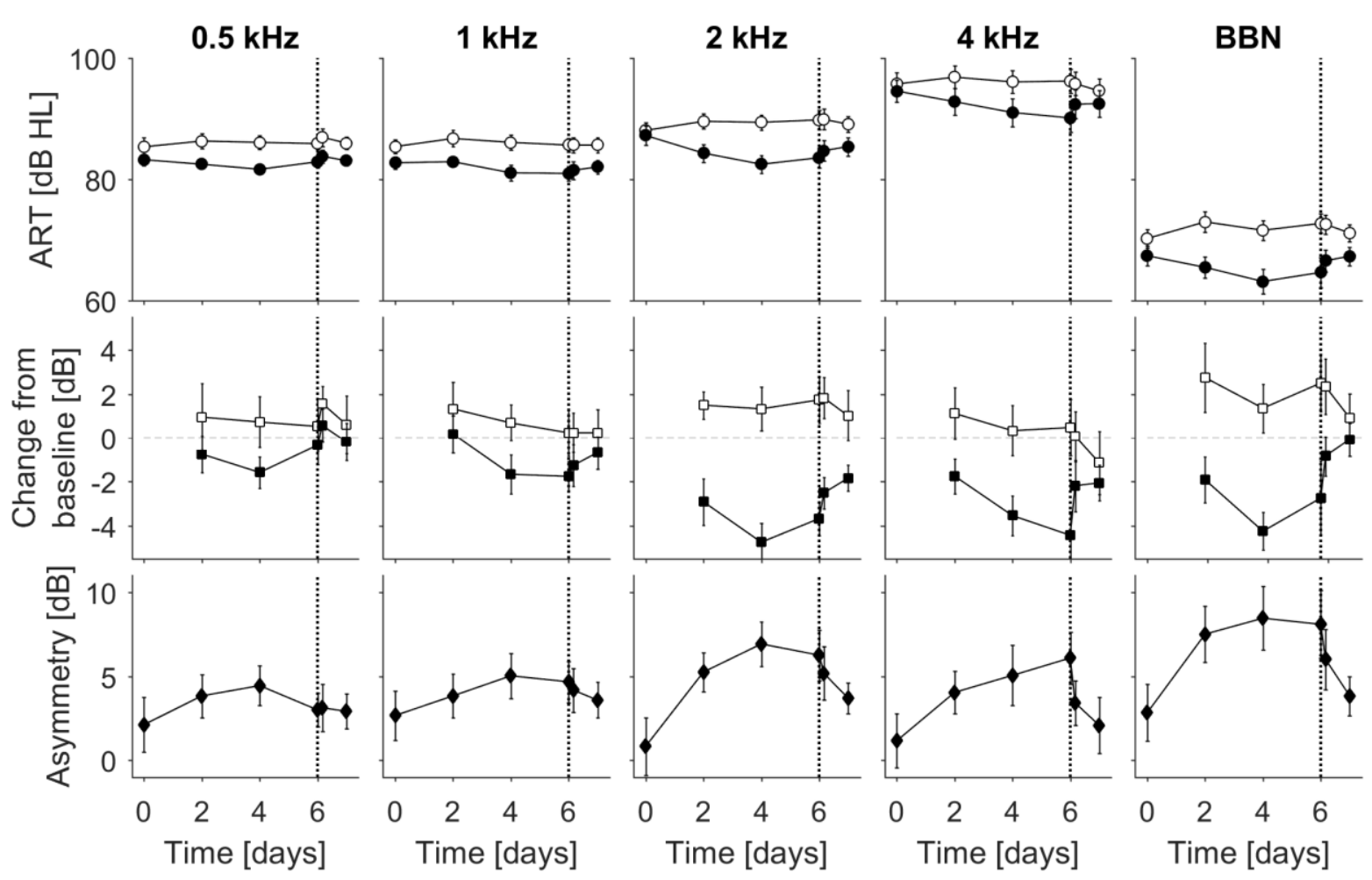

Fig. 2. Time course of changes in ARTs during 6 days of earplug use, and 4 and $24 \mathrm{~h}$ after

840 removal of the earplug. ARTs were elicited with pure tones $(0.5,1,2$, or $4 \mathrm{kHz})$ or broadband

841 noise (BBN). The top row shows the mean ARTs from the treatments ears (filled circles) and

842 the control ears (open circles). In the middle row, changes from the pre-earplug baseline

843 values at day 0 are shown for the control (open squares) and the plugged ears (filled squares).

844 The bottom row shows the development of the asymmetry in ART between the ears (control

$845-$ treatment) over time. The vertical dotted lines indicate the time point at which the earplug

846 was removed (day 6$)$. Errors bars show \pm 1 standard deviation $(n=24)$. 

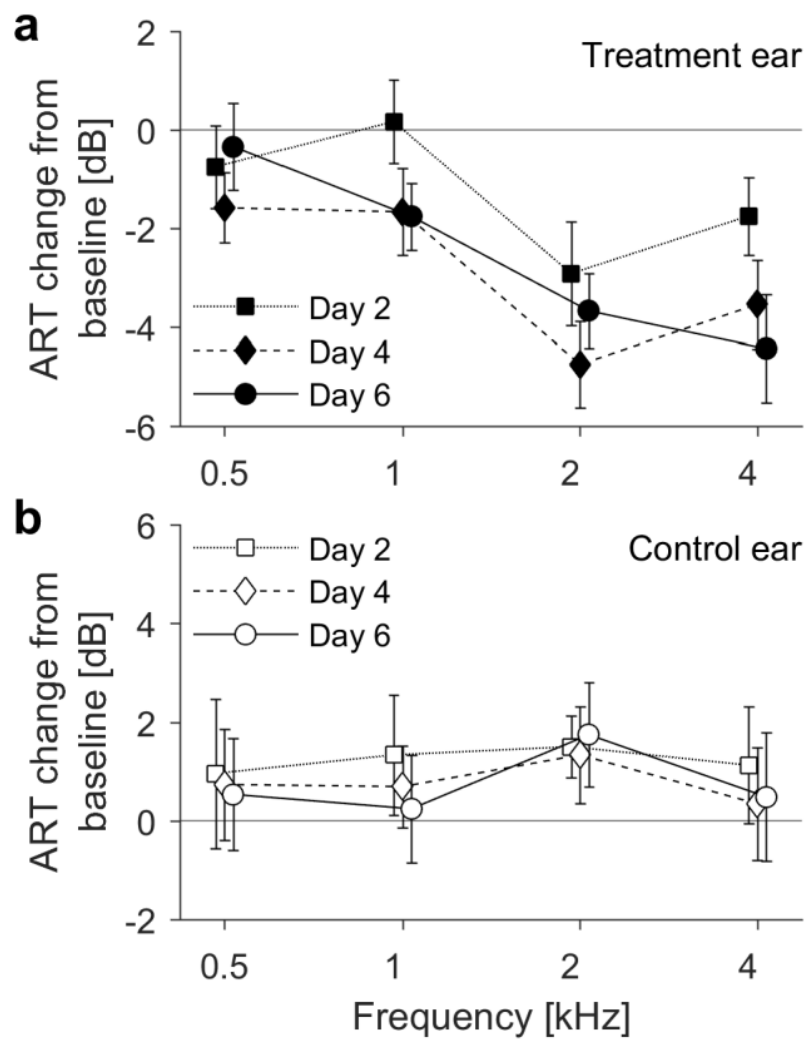

Fig. 3. Frequency-specificity of earplug-induced changes in ARTs. a) Changes in ipsilateral

850 ARTs from pre-earplug baseline in the treatment ear at day 2 (squares with dotted line), day 4

851 (diamonds with dashed line), and day 6 (circles with solid line). b) Changes in ipsilateral 852 ARTs in the control ear, line styles as in (a). Errors bars show \pm 1 standard deviation $(n=24)$. 

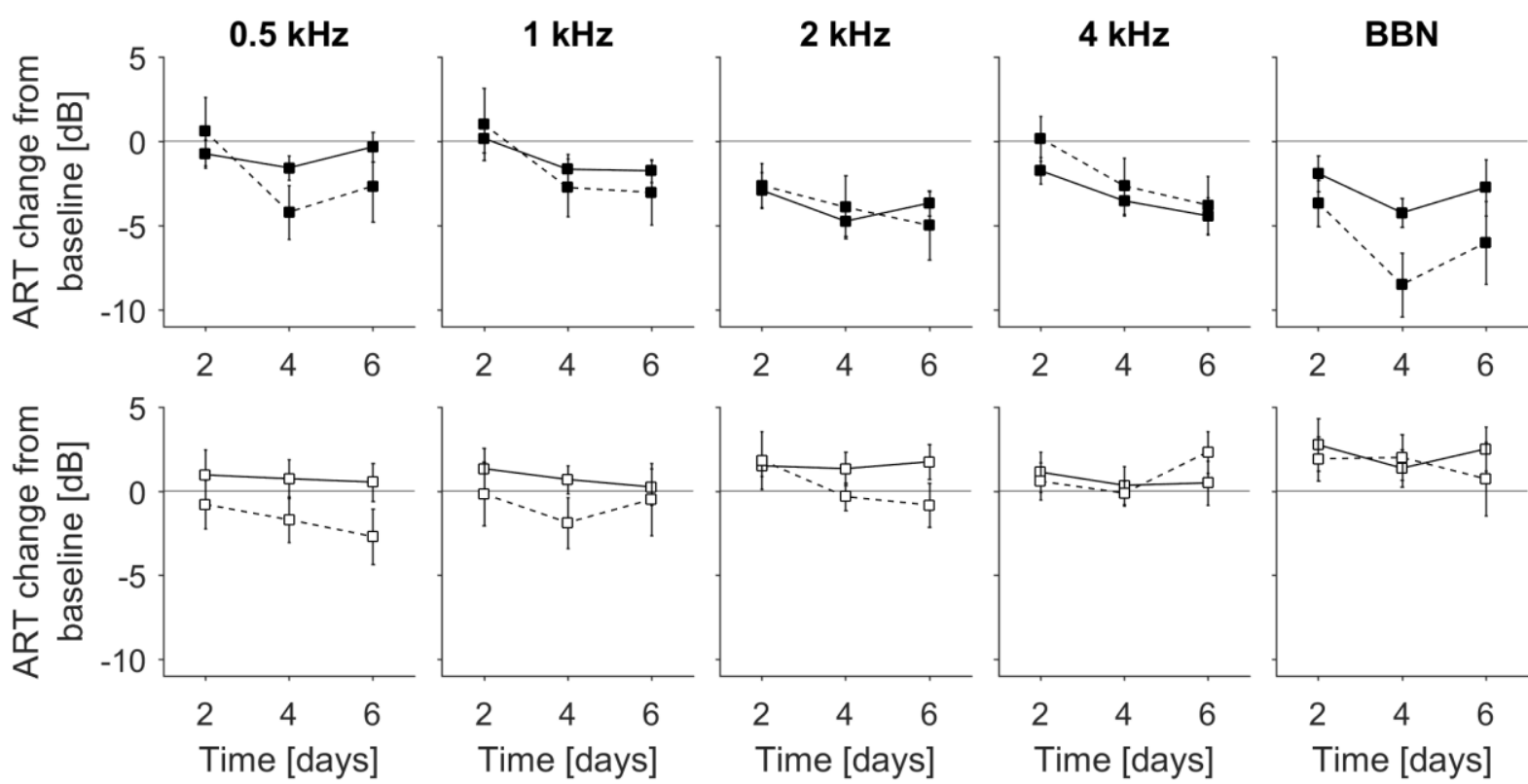

Fig. 4. Changes in ipsi- and contralateral ARTs after auditory deprivation through an earplug.

856 All graphs show changes from the pre-earplug baseline at day 0. Solid lines denote

857 measurements where the ART was measured ipsilateral to the presentation of the eliciting 858 stimulus, dashed lines show results for contralateral ART measurements. The top row shows 859 ART changes for presentation of the eliciting stimuli to the treatment ear, and the bottom row 860 for presentation to the control ear. Errors bars show \pm 1 standard deviation $(n=24)$. 